\title{
Ressecção de endometriose intestinal com dupla ressecção discoide com grampeador circular: relato de caso
}

\section{Intestinal endometriosis with double discoid resection using a circular stapler: case report}

Flora Cruz de Almeida ${ }^{1}$. Thaís Fontes de Magalhães ${ }^{1}$. Letícia Matoso Freire ${ }^{1}$. Kathiane Lustosa Augusto ${ }^{2}$. Manuela Cavalcante Portela Marinho ${ }^{3}$. Carla Camila Rocha Bezerra ${ }^{4}$. Sthela Maria Murad Regadas ${ }^{5}$. Lusmar Veras Rodrigues ${ }^{6}$. Leonardo Robson Pinheiro Sobreira Bezerra ${ }^{7}$.

1 Acadêmica do curso de Medicina, Universidade Federal do Ceará (UFC), Fortaleza, Ceará, Brasil. 2 Mestre em Ciências MédicoCirurgicas, Universidade Federal do Ceará (UFC), Fortaleza, Ceará, Brasil. 3 Residência Médica em Ginecologia e Obstetrícia, Hospital Geral de Fortaleza (HGF), médica da Maternidade Escola Assis Chateaubriand (MEAC), Fortaleza, Ceará, Brasil. 4 Residente de Coloproctologia, Hospital Universitário Walter Cantídio (HUWC), Fortaleza, Ceará, Brasil. 5 Pós-doutorado na Cleveland Clinic, Flórida, Estados Unidos da América. Professora associada do Departamento de Cirurgia da Universidade Federal do Ceará (UFC), Fortaleza, Ceará, Brasil. 6 Doutor em Medicina pela Universidade Federal do Ceará (UFC). Professor do Departamento de Cirurgia da Universidade Federal do Ceará, Fortaleza, Ceará, Brasil. 7 Professor do Departamento Materno Infantil da Universidade Federal do Ceará (UFC), Fortaleza, Ceará, Brasil. Doutor em Ginecologia, Universidade Federal de São Paulo (UNIFESP), São Paulo, São Paulo, Brasil.

\section{RESUMO}

A endometriose é uma doença inflamatória que pode cursar com uma variedade de sintomas álgicos pélvico-abdominais e com infertilidade. As lesões intestinais normalmente possuem um caráter mais agressivo que as de demais localizações, o que as faz serem, muitas vezes, refratárias ao tratamento conservador, tornando a ressecção cirúrgica das lesões a melhor opção. A técnica cirúrgica a ser usada objetiva a remoção completa dos endometriomas, a restauração da anatomia e a preservação da função orgânica. A cirurgia minimamente invasiva por laparoscopia é uma alternativa adequada para reduzir a morbidade associada aos procedimentos abertos tradicionais. O subtipo de endometriose com acometimento intestinal constitui desafio terapêutico mais complexo. As opções no tratamento cirúrgico incluem: shaving do nódulo de endometriose intestinal, ressecção discoide do nódulo por grampeador circular e ressecção segmentar intestinal com anastomose término-terminal. O objetivo deste relato é demonstrar a viabilidade técnica de ressecção de endometriose intestinal com dupla ressecção discoide com grampeador circular em paciente em que se realizou laparoscopia para tratamento de endometriose pélvica.

Palavras-chave: Endometriose. Laparoscopia. Mulheres. Cirurgia videoassistida.

\section{ABSTRACT}

Endometriosis is an inflammatory disease that may be associated with a variety of abdominal pain symptoms and infertility. Intestinal lesions are typically more aggressive than in other locations. They often show unsatisfactory responses to conservative treatments, thus making surgery the best treatment choice. The surgical techniques used aim for the complete removal of endometriotic lesions while restoring the anatomy and preserving organ function. Minimally invasive laparoscopic surgery is a suitable alternative to reduce the morbidity associated to traditional open procedures. The subtype of endometriosis with bowel involvement is complex therapeutic challenge. Surgical treatment options include: shaving of intestinal endometriosis nodule, discoid resection of nodule with a circular stapler and segmental resection with end-to-end anastomosis. The objective of this report is to demonstrate the technical feasibility of intestinal endometriosis resection with double discoid resection using a circular stapler in a patient who underwent laparoscopy for treatment of pelvic endometriosis.

Keywords: Endometriosis. Laparoscopy. Women. Video-assisted surgery.

Autor correspondente: Flora Cruz de Almeida, Rua Armando Oliveira, 261, Parquelândia, Fortaleza, Ceará. CEP: 60450-060.Telefone: +55 85 99680-9718. E-mail: floracruzdealmeida@yahoo.com.br

Conflito de interesses: Não há qualquer conflito de interesses por parte de qualquer um dos autores.

Recebido em: 29 Out 2015; Revisado em: 10 Jan 2016; Aceito em: 12 Fev 2016. 


\section{INTRODUÇÃO}

A endometriose é uma doença inflamatória, caracterizada pela presença de glândulas e estroma endometriais em localizações ectópicas, principalmente no peritônio pélvico, nos ovários e no septo reto-vaginal. As pacientes podem apresentar uma grande variedade de sintomas álgicos pélvico-abdominais e infertilidade. ${ }^{1}$ As opções de tratamento são variadas, desde terapias analgésicas e hormonais até cirurgia minimamente invasiva de ressecção de lesões invasivas. ${ }^{2}$

Um dos seus principais desafios terapêuticos é a endometriose com acometimento intestinal. O intestino é mais comumente atingido pela endometriose profunda infiltrativa (EPI). Tais lesões possuem um caráter mais agressivo que as demais, o que as tornam, muitas vezes, refratárias ao tratamento farmacológico, tornando a ressecção cirúrgica das lesões a melhor opção. ${ }^{3,4}$

A técnica cirúrgica a ser usada objetiva a remoção completa da doença, restauração da anatomia e preservação da função orgânica. A cirurgia minimamente invasiva por laparoscopia é uma alternativa adequada para reduzir a morbidade associada aos procedimentos "abertos" tradicionais. As opções no tratamento cirúrgico incluem: shaving do nódulo de endometriose intestinal, ressecção discoide do nódulo de endometriose por grampeador circular e ressecção segmentar com anastomose término-terminal.

O objetivo desse relato é demonstrar a viabilidade técnica de ressecção de endometriose intestinal com dupla ressecção discoide com grampeador circular em paciente onde se realizou laparoscopia para tratamento de endometriose pélvica. Esta técnica é apresentada aqui como opção à ressecção segmentar do intestino, que apresenta relativa alta taxa de morbidade em casos de lesões endometrióticas maiores e sintomáticas.

\section{RELATO}

Paciente V.N.A, 33 anos, com história de dismenorreia cíclica, progressiva, dispareunia profunda, dor pélvica crônica desde a menarca, disquezia, sangramento intestinal e diarreia associada à menstruação. A dor a impossibilitava de continuar suas atividades laborais de vendedora. A ultrassonografia transvaginal para mapeamento de endometriose com preparo intestinal mostrou achados compatíveis com comprometimento endometriótico profundo, associada à lesão de $4 \mathrm{~cm}$ em sigmoide, localizada $12 \mathrm{~cm}$ proximal à margem anal. Procedeu-se à laparoscopia com equipe multidisciplinar (ginecologia e coloproctologia). Obtivemos o achado de lesão em parede anterior de retossigmóide aderida a torus uterino e ligamentos uterossacros direito e esquerdo. Procedeu-se à ureterólise bilateral, identificação dos nervos hipogástricos direito e esquerdo e dissecção dos espaços pararretais direito e esquerdo. Seguiu-se à identificação das artérias uterinas bilateralmente e seus cruzamentos com os ureteres. Realizouse dissecção da lesão nodular em fórnice vaginal com exérese da mesma por colpotomia no fundo de saco vaginal. Seguida de sutura da abertura vaginal. Após a secção do espaço retovaginal, identificou-se lesão intestinal, $12 \mathrm{~cm}$ da margem anal, de aproximadamente $4 \mathrm{~cm}$. A técnica de shaving foi aplicada à lesão intestinal com ressecção parcial de seu volume. Uma vez observado significativo remanescente de lesão em parede intestinal procedeu-se à tentativa de ressecção da lesão por grampeador circular aplicado por via transretal. Para tanto, procedeu-se inicialmente à individualização da área de lesão em parede retal anterior com dois pontos de sutura simples à montante e à jusante da lesão. Utilizou-se fio de polipropileno zero com sutura no sentido perpendicular à taenia. Após a passagem dos fios a área de lesão é individualizada e acomodada no grampeador circular. Observou-se, novamente, presença ainda de significativo remanescente da lesão. Após avaliar-se possibilidade de manutenção da permeabilidade da luz e da mobilidade do reto submeteu-se à um novo grampeamento circular, também pela via transretal, desta feita utilizou-se um grampeador de menor calibre. Após o novo procedimento finalmente constatouse exérese completa da lesão. Após o duplo grampeio, foi realizado "teste do borracheiro" para avaliar vazamentos, cujo resultado foi negativo.

\section{DISCUSSÃO}

Entre 5 a $27 \%$ das mulheres com endometriose apresentam EPI com infiltração intestinal - que não só causa maior impacto sobre a dor, como tem uma alta incidência de morbidade cirúrgica. $^{5}$

O tratamento da endometriose intestinal pode ser conservador (clínico) ou cirúrgico, com ressecção dos focos da doença. $\mathrm{O}$ tratamento cirúrgico é indicado àquelas que não melhoraram com uma tentativa farmacológica ou estão sob suspeita de obstrução. ${ }^{2}$ Além disso, hoje, não há tratamento farmacológico comprovado para uso em longo prazo, e os disponíveis atualmente não têm durabilidade de eficácia após a descontinuação da terapia. ${ }^{6}$

No caso da EPI, o tratamento hormonal induz uma melhoria inicial temporária, mas o problema ressurge mesmo com o uso contínuo da medicação. Diante disso, o tratamento cirúrgico com ressecção completa da endometriose profunda tem sido a recomendação terapêutica com melhores resultados para o alívio sintomático em longo prazo. ${ }^{7}$ Não existe consenso em relação à técnica cirúrgica ideal para abordar a endometriose intestinal, mas a excisão deve ser completa, a fim de alcançar o máximo alívio álgico e a mínima taxa de recorrências. ${ }^{7,8}$

Existem diversas vias de acesso à cavidade pélvica, como a laparoscopia, laparotomia ou via vaginal. A laparoscopia e a laparotomia são igualmente eficazes, porém a primeira está relacionada com melhor período pós-operatório, menor tempo de internação e reabilitação, além de melhor resultado estético. ${ }^{9}$ Já a via vaginal é limitada pois proporciona um acesso difícil às lesões. ${ }^{10}$

No caso descrito, a via de acesso foi laparoscópica e a técnica cirúrgica utilizada chama-se dupla ressecção discoide com grampeador circular. A ressecção circular, ou em disco, é 
indicada idealmente para a exérese de nódulos endometrióticos com diâmetro inferior a $3 \mathrm{~cm}$ que acometem a parede retal. Após a identificação da lesão e seu isolamento, o grampeador circular é inserido por via anal. Laparoscopicamente acomoda-se toda a lesão dentro do grampeador e realiza-se seu fechamento e disparo. Assim, pode-se observar a exérese apenas da parede retal anterior que engloba o nódulo. ${ }^{8,11}$

A técnica usada visa combater a alta taxa de morbidade ainda presente nesse tipo de cirurgia. O tempo operatório médio de sua realização é menor em comparação com a retossigmoidectomia e o número de fístulas que aparecem no pós-operatório é baixo, o que a torna uma técnica promissora e alternativa à ressecção segmentar. ${ }^{12,13}$ É válido pontuar que a ressecção discoide com grampeador circular já foi criticada por apresentar um alto percentual de lesão residual no estudo histológico, enquanto outros autores negam tal afirmativa e apresentaram bons resultados. ${ }^{14}$

\section{REFERÊNCIAS}

1. Burney RO, Giudice LC. Pathogenesis and pathophysiology of endometriosis. Fertil Steril. 2012;98(3):511-9.

2. Kennedy S, Bergqvist A, Chapron C, D'Hooghe T, Dunselman G, Greb R, et al. ESHRE guideline for the diagnosis and treatment of endometriosis. Hum Reprod. 2005;20:2698-704.

3. Cornillie FJ, Oosterlynck D, Lauweryns JM, Koninckx PR. Deeply infiltrating pelvic endometriosis: histology and clinical significance. Fertil Steril. 1990;53(6):978-83.

4. Sagae UE, Lima DM, Cavalli N, Sagae LM, Tanaka TM, Bonatto MW, et al. Importância da ultra-sonografia anorretal tridimensional na decisão terapêutica da endometriose profunda. Rev bras coloproct. 2009;29(4):435-42.

5. Abrão MS, Podgaec S, Dias JA Jr, Averbach M, Silva LF, Marino de Carvalho F. Endometriosis lesions that compromise the rectum deeper than the inner muscularis layer have more than $40 \%$ of the circumference of the rectum affected by the disease. J Minim Invasive Gynecol. 2008;15(3):280-5.

6. Fuldeore M, Hongbo Yang H, Du EX, Soliman AM, Wu EQ, Winkel C. Healthcare utilization and costs in women diagnosed with endometriosis before and after diagnosis: a longitudinal analysis of claims databases. Fertility and Sterility. 2015;103(1):163-171.

7. Costa LM, Ávila I, Filogonio ID, Machado LG; Carneiro MM. Tratamento laparoscópico de 98 pacientes com endometriose intestinal. Rev bras coloproct. 2010;30(1):31-36.

8. De Cicco C, Corona R, Schonman R, Mailova K, Ussia A,
Observa-se que a paciente tem mais de 30 anos, o que diminui o risco de necessidade de cirurgia de repetição, e está em sua primeira operação, que, por si só, tende a produzir uma resposta melhor do que os procedimentos cirúrgicos posteriores. ${ }^{15}$

\section{CONCLUSÃO}

$\mathrm{Na}$ técnica de dupla ressecção discoide com grampeador circular por ocorrer exérese apenas da parede retal anterior que engloba o nódulo, observa-se resultados mais funcionais se comparado a pacientes que se submetem a excisão radical do segmento intestinal acometido e diminuição da morbidade associada à retossigmoidectomia. A técnica cirúrgica de dupla ressecção discoide com grampeador circular apresenta, portanto, uma alternativa à ressecção segmentar em pacientes selecionados com endometriose intestinal.

Koninckx PR. Bowel resection for deep endometriosis: a systematic review. BJOG. 2011;118(3):285-91.

9. Fayez, JA, Collazo LM. Comparison between laparotomy and operative laparoscopy in the treatment of moderate and severe stages of endometriosis. Int J Fertil. 1990;35(5):272-9.

10. Dunselman GA, Vermeulen N, Becker C, Calhaz-Jorge C, D'Hooghe T, De Bie B, et al. ESHRE guideline: management of women with endometriosis. Hum Reprod. 2014;29(3):400-12.

11. Fleisch MC, Xafis D, De Bruyne F, Hucke J, Bender HG, Dall P. Radical resection of invasive endometriosis with bowel or bladder involvement--long-term results. Eur J Obstet Gynecol Reprod Biol. 2005;123(2):224-9.

12. Oliveira MA, Crispi CP, Oliveira FM, Reis PS Jr, Raymundo TS, Pereira TD. Double circular stapler technique for bowel resection in rectosigmoid endometriosis. J Minim Invasive Gynecol. 2014;21(1):136-41.

13. Kondo W, Ribeiro R, Zomer MT, Hayashi R. Laparoscopic double discoid resection with a circular stapler for bowel endometriosis. J Minim Invasive Gynecol. 2015;22(6):929-31.

14. Costa LM, Ávila I, Filogonio ID, Machado LG, Carneiro MM. Tratamento laparoscópico de 98 pacientes com endometriose intestinal. Rev bras coloproct. 2010;30(1):31-36.

15. Johnson NP, Hummelshoj L; World Endometriosis Society Montpellier Consortium. Consensus on current management of endometriosis. Hum Reprod. 2013;28(6):1552-68.

\section{Como citar:}

Almeida FC, Magalhães TF, Freire LM, Augusto KL, Marinho MC, Bezerra CC, et al. Ressecção de endometriose intestinal com dupla ressecção discoide com grampeador circular: relato de caso. Rev Med UFC. 2016 jan-jun;56(1):52-54. 